\title{
The expression of trefoil factor 3 is related to histologic subtypes and invasiveness in lung adenocarcinoma
}

\author{
WENJUAN LUO ${ }^{1,2^{*}}$, SHINICHIRO TAHARA ${ }^{1 *}$, KEISUKE KAWASAKI ${ }^{1}$, \\ AYAKA KOBAYASHI $^{1}$, SATOSHI NOJIMA $^{1}$ and EIICHI MORII ${ }^{1}$ \\ ${ }^{1}$ Department of Pathology, Osaka University Graduate School of Medicine, Osaka 565-0871, Japan; \\ ${ }^{2}$ School of Pharmacy, Health Science Center, Xi'an Jiaotong University, Xi'an, Shaanxi 710061, P.R. China
}

Received July 3, 2019; Accepted October 8, 2019

DOI: $10.3892 / 01.2020 .12325$

\begin{abstract}
Adenocarcinoma is the most common histological type of lung cancer and has various histologic subtypes, including lepidic, papillary, acinar and invasive mucinous adenocarcinoma. Histologic subtypes are associated with tumor invasiveness. For example, the lepidic subtype is less invasive than the papillary/acinar subtype. Trefoil factor 3 (TFF3) is a small secreting protein that is a member of the trefoil factor family, which is involved in mucosal stabilization and repair through its mitogenic and antiapoptotic activities. TFF3 overexpression is associated with various types of cancer. In lung cancer, TFF3 is expressed significantly in adenocarcinoma. However, the relationship between TFF3 expression and histologic subtypes in lung adenocarcinoma is unclear. The current study immunohistochemically revealed that, beside invasive mucinous carcinoma, the expression of TFF3 in papillary and acinar adenocarcinoma was significantly higher than that in lepidic adenocarcinoma. To further confirm these results, the expression of TFF3 in cases with both lepidic and papillary/ acinar areas were examined. The expression of TFF3 in papillary/acinar areas was significantly higher when compared with lepidic areas in a single sample. Furthermore, using the lung adenocarcinoma cell line A549, TFF3-knockdown cells were generated. The results revealed that knockdown of TFF3 attenuated invasion. In vitro and immunohistochemical assays using clinical samples demonstrated that TFF3 expression was associated with lung adenocarcinoma invasiveness. To the best of our knowledge, the current study is the first to report that TFF3 expression was associated with the histologic subtypes of lung adenocarcinoma.
\end{abstract}

Correspondence to: Professor Eiichi Morii, Department of Pathology, Osaka University Graduate School of Medicine, 2-2 Yamada-oka, Suita, Osaka 565-0871, Japan

E-mail: morii@molpath.med.osaka-u.ac.jp

*Contributed equally

Key words: trefoil factor 3, lung adenocarcinoma, immunohistochemistry, invasiveness

\section{Introduction}

Lung cancer is the leading cause of cancer-related mortality. Non-small cell lung carcinoma accounts $85 \%$ of lung cancer, and adenocarcinoma is the most common histological type (1). Lung adenocarcinoma has various histologic subtypes, such as lepidic, acinar, papillary, micropapillary, solid, invasive mucinous adenocarcinoma, and so on. Histologic subtypes are a prognostic factor; lepidic subtype harbors the best prognostic course, whereas micropapillary and solid patterns have a more aggressive behavior. Moreover, most lung adenocarcinomas demonstrate a mixture of different histologic patterns. The combination of histologic subtypes is important for prognosis (2).

Trefoil factor 3 (TFF3) is a small secreting protein and a member of trefoil factor family, which is involved in mucosal stabilization and repair through mitogenic and antiapoptotic activities $(3,4)$. TFF3 is distributed mainly in goblet cells of intestine and lung (5). Overexpression of TFF3 has been reported to be associated with several types of cancer, such as stomach, uterus, and breast (6-8). In lung cancer, TFF3 is expressed significantly in adenocarcinoma and is a useful biomarker to distinguish between adenocarcinoma and squamous cell carcinoma (9). However, the relationship between TFF3 expression and histologic subtypes in lung adenocarcinoma has not been examined. Here we revealed the relationship between TFF3 expression and histologic subtypes in lung adenocarcinoma. By immunohistochemical analysis of 93 lung adenocarcinoma cases, we showed TFF3 was highly expressed not only in invasive mucinous adenocarcinoma but also in papillary and acinar adenocarcinoma. The expression level of TFF3 was higher in papillary/acinar subtype than in lepidic subtype; the former was more aggressive subtype than the latter. Moreover, we generated TFF3-knockdown lung adenocarcinoma cells and showed that the depletion of TFF3 attenuated invasion. TFF3 expression is correlated to invasiveness in lung adenocarcinoma.

\section{Materials and methods}

Patients. We examined 93 cases undergoing surgery for adenocarcinoma of the lung at Osaka University Hospital from 2013 to 2018. No prior therapy was administered in any 
case. Due to the short observation period, only 3 cases died of the underlying disease, and then we focused the relation of TFF3 expression to histological subtypes rather than prognosis. Histologic subtypes were classified according to WHO criteria (10). The histological subtypes were lepidic $(n=20)$, acinar $(n=15)$, papillary $(n=22)$, solid adenocarcinoma $(n=19)$, and invasive mucinous adenocarcinoma $(n=17)$ (Table I). Lepidic adenocarcinoma included 3 cases of adenocarcinoma in situ (AIS) and 3 cases of minimally invasive adenocarcinoma (MIA). Resected specimens were fixed in 10\% formalin and processed for paraffin embedding. Specimens were stored at room temperature in a dark room. Specimens for evaluation were sectioned at $4 \mu \mathrm{m}$ thickness and stained with hematoxylin and eosin (H\&E). The study was approved by the Ethical Review Board of the Graduate School of Medicine, Osaka University (approval no. 16293). Informed consent was obtained from all patients.

Immunohistochemistry for TFF3 and evaluation with histological score (H-score). Expression of TFF3 was examined with the primary rabbit anti-TFF3 monoclonal antibody (dilution 1:2,000 cat. no. ab108599; Abcam). Subsequent to deparaffinization with xylene and rehydration with graded alcohol treatment, sections were heated to $121^{\circ} \mathrm{C}$ in the Pascal Pressurized Heating Chamber (Agilent Technologies, Inc.). After cooling, the sections were washed in phosphate-buffered saline, blocked with blocking solution (cat. no. X0909; Agilent Technologies, Inc.) and incubated with anti-TFF3 antibody. Next, the sections were treated with a ChemMate EnVision kit (Agilent Technologies, Inc.) that contains a polymerized secondary antibody to increase detection sensitivity for the primary antibody. Diaminobenzidine (DAB) (Agilent Technologies, Inc.) was used as a chromogen. Sections were counterstained with hematoxylin and observed by microscopy. As the negative control, staining was carried out in the absence of primary antibody. Staining intensity $(0$, $1+, 2+$ or $3+$ ) was determined for each sample independently by two pathologists (S.T. and E.M.). H-score was calculated using the following formula: [1x (\% tumor cells of $1+)+2 \mathrm{x}$ $(\%$ tumor cells of $2+)+3 x(\%$ tumor cells of $3+)]$.

Cell line. The human lung adenocarcinoma cell line, A549 was obtained from ATCC. Cells were cultured in DMEM supplemented with $10 \%$ FBS (Biosera) and in a humidified $5 \% \mathrm{CO}_{2}$ incubator at $37^{\circ} \mathrm{C}$.

Immunoblotting. Cells were lysed in buffer containing $10 \mathrm{mM}$ 4-(2-hydroxyethyl)-1-piperazineethanesulfonic acid, $10 \mathrm{mM}$ $\mathrm{KCl}, 1 \mathrm{mM}$ ethylenediaminetetraacetic acid, $1 \mathrm{mM}$ dithiothreitol and $0.1 \%$ Nonidet P-40. Electrophoresis was performed in $5-20 \%$ gradient sodium dodecyl sulphate-polyacrylamide gels (ATTO), and proteins were transferred to polyvinylidene fluoride membranes (Merck KGaA). We used the primary anti-TFF3 antibody at 1:500 and it was detected using a horseradish peroxidase-conjugated anti-rabbit $\mathrm{IgG}(\mathrm{H}+\mathrm{L}$ chain) $(1: 5,000$; MBL). We quantified the results using ImageJ (https://imagej. nih.gov/ij/).

Generation of TFF3-knockdown cells using siRNA-mediated silencing. A549 cells $\left(1 \times 10^{5}\right)$ seeded into six-well culture plates were transfected with TFF3-targeting siRNA (Silencer Select s14039, s14040 and s14041; Thermo Fisher Scientific, Inc.) or non-targeting control siRNA (AM4611; Thermo Fisher Scientific, Inc.) using Lipofectamine RNAiMAX Reagent (Thermo Fisher Scientific, Inc.) at a final concentration of $50 \mathrm{nM}$. Cells were subjected to the immunoblotting analysis and matrigel invasion assay $72 \mathrm{~h}$ after siRNA transfection.

Matrigel invasion assay. Tumor cell invasion was examined using the Corning BioCoat Matrigel Invasion Chamber (Corning, Inc.). Tumor cells were placed in the upper chamber in DMEM without FBS and incubated at $37^{\circ} \mathrm{C}$ for $24 \mathrm{~h}$. The lower chamber contained DMEM with $10 \%$ FBS. Invasive cells, which migrated to the lower side of the upper chamber, were stained with Diff-Quik (Sysmex). The number of invasive cells was counted in five random fields per chamber at high magnification.

Statistical analysis. Statistical analyses were performed using JMP Pro v14 software (SAS Institute Inc.). Results were shown as the means \pm standard error (SE). Differences in results were determined using Student's t-test, Wilcoxon signed-rank test, and analysis of variance (ANOVA) followed by Dunnett's test. $\mathrm{P}<0.05$ was considered to indicate a statistically significant difference.

\section{Results}

Expression of TFF3 with immunohistochemical analysis. TFF3 was hardly expressed in non-cancerous alveolar epithelial cells (Fig. 1A), but strongly in normal bronchial glands and bronchial epithelium (Fig. 1B). Lung adenocarcinoma cells expressed TFF3 in their cytoplasm, the expression level of which was various among cases. The typical staining patterns were shown in Fig. 1C-E; weakly in Fig. 1C, moderately in Fig. 1D, and strongly in Fig. 1E.

Association between TFF3 expression and histologic subtypes. The associations between TFF3 expression level (H-score) and histologic subtypes were evaluated (Table II). The expression of TFF3 was hardly detected in lepidic subtype (Fig. 2A), moderately in papillary and acinar subtypes (Fig. 2B and C), and weakly in solid subtype (Fig. 2D). The highest expression level of TFF3 was detected in invasive mucinous carcinoma (Fig. 2E), in which tumor cells showed diffuse and strong positivity. The rank order of TFF3 expression level was as follows; invasive mucinous adenocarcinoma > papillary and acinar subtypes $>$ solid and lepidic subtypes.

Several lung adenocarcinoma is known to be composed of mixture of histologic subtypes. In fact, 38 cases showed the mixture of lepidic subtype and papillary/acinar subtype (Fig. 3A; boxed area with solid line was lepidic subtype, whereas boxed area with dotted line was papillary subtype). Then, we compared TFF3 expression level between area of lepidic subtype (Fig. 3B) and that of papillary/acinar subtype (Fig. 3C) in an individual case. H-score of papillary/acinar area was significantly higher than that of lepidic area (Fig. 3D).

Involvement of TFF3 in the invasion of lung adenocarcinoma cells. We transfected A549 cells with 3 individual siRNA 
Table I. Histologic subtypes of 93 cases of lung adenocarcinoma.

Histologic subtypes

Number of patients

Lepidic adenocarcinoma ${ }^{a}$

20

Acinar adenocarcinoma

15

Papillary adenocarcinoma

22

Solid adenocarcinoma

19

Invasive mucinous adenocarcinoma

17

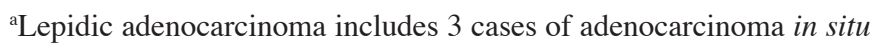
and 3 cases of minimally invasive adenocarcinoma.

Table II. TFF3 expression in various histologic subtypes of lung adenocarcinoma.

\begin{tabular}{lc}
\hline Histologic subtype & H-score $^{\mathrm{a}}$ \\
\hline Lepidic adenocarcinoma & $12.95 \pm 4.94$ \\
Acinar adenocarcinoma & $30.87 \pm 9.26^{\mathrm{b}}$ \\
Papillary adenocarcinoma & $36.05 \pm 10.74^{\mathrm{b}}$ \\
Solid adenocarcinoma & $18.47 \pm 6.58$ \\
Invasive mucinous adenocarcinoma & $137.35 \pm 18.33^{\mathrm{b}}$ \\
\hline
\end{tabular}

${ }^{\mathrm{a} D}$ Data are presented as the mean \pm standard error. ${ }^{\mathrm{b}} \mathrm{P}<0.05$ vs. lepidic adenocarcinoma. TFF3, Trefoil factor 3 .

duplexes specific for TFF3 (siTFF3 \#1, \#2 and \#3), or a nontargeting control siRNA (siControl), and confirmed the decrease in TFF3 protein expression in TFF3-knockdown cells (Fig. 4A). We found that in comparison with control cells, the invasion of TFF3-knockdown cells was attenuated (Fig. 4B). Thus, TFF3 is involved in the invasion of lung adenocarcinoma cells.

\section{Discussion}

TFF3 is related to mucosal stabilization in gastrointestinal tract in normal condition and is upregulated in various types of cancer (4,6-8). To date, no studies have compared TFF3 expression in histologic subtypes of lung adenocarcinoma. In the present study, we pointed that the expression level of TFF3 in invasive mucinous carcinoma was the highest, followed by papillary, acinar, solid, and lepidic subtypes of adenocarcinoma. It's not surprising that the expression of TFF3 in invasive mucinous carcinoma was overwhelmingly high because TFF3 is mainly distributed in mucous cells in normal condition. Besides invasive mucinous carcinoma, the expression of TFF3 in papillary and acinar adenocarcinoma was significantly higher than in lepidic adenocarcinoma. We considered that TFF3 was related to invasiveness in lung adenocarcinoma. To confirm that, we used 38 cases with both lepidic and papillary/acinar areas. A subset of lung adenocarcinoma follows a linear multistep progression, in which a precursor lesion progresses to adenocarcinoma in situ, which is followed by invasive adenocarcinoma (11). In the
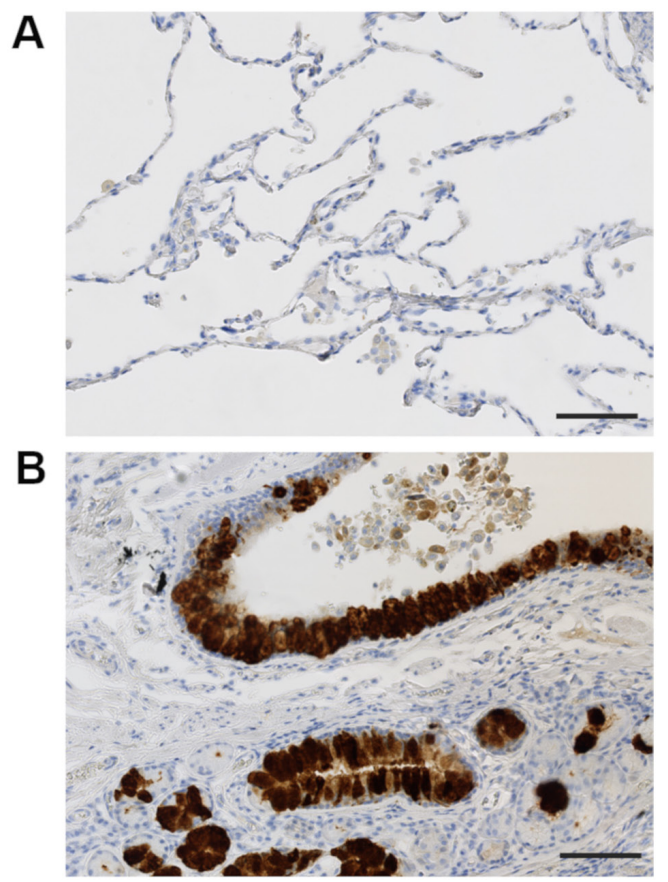

C

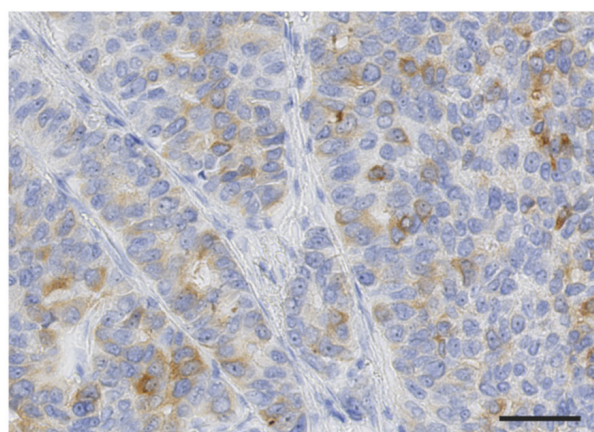

D

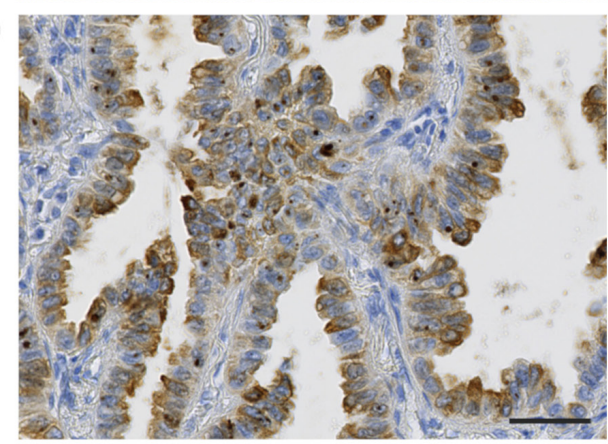

E

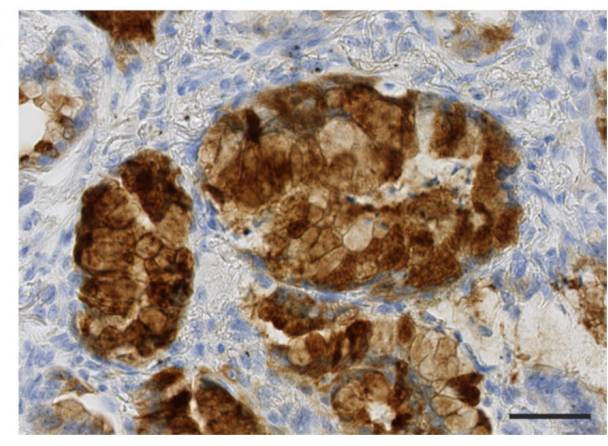

Figure 1. Varying immunohistochemical staining intensities demonstrate TFF3 expression in normal lung and lung adenocarcinoma. (A) Non-cancerous alveolar epithelial cells hardly expressed TFF3. (B) Normal bronchial glands and bronchial epithelium strongly expressed TFF3. (C-E) Representative immunohistochemistry images of lung adenocarcinoma were presented with intensity scores of weak $(1+; \mathrm{C})$, moderate $(2+; \mathrm{D})$ and strong $(3+; \mathrm{E})$, respectively. Scale bars in (A and B), $100 \mu \mathrm{m}$; scale bares in (C-E), $50 \mu \mathrm{m}$. TTF3, trefoil factor 3. 

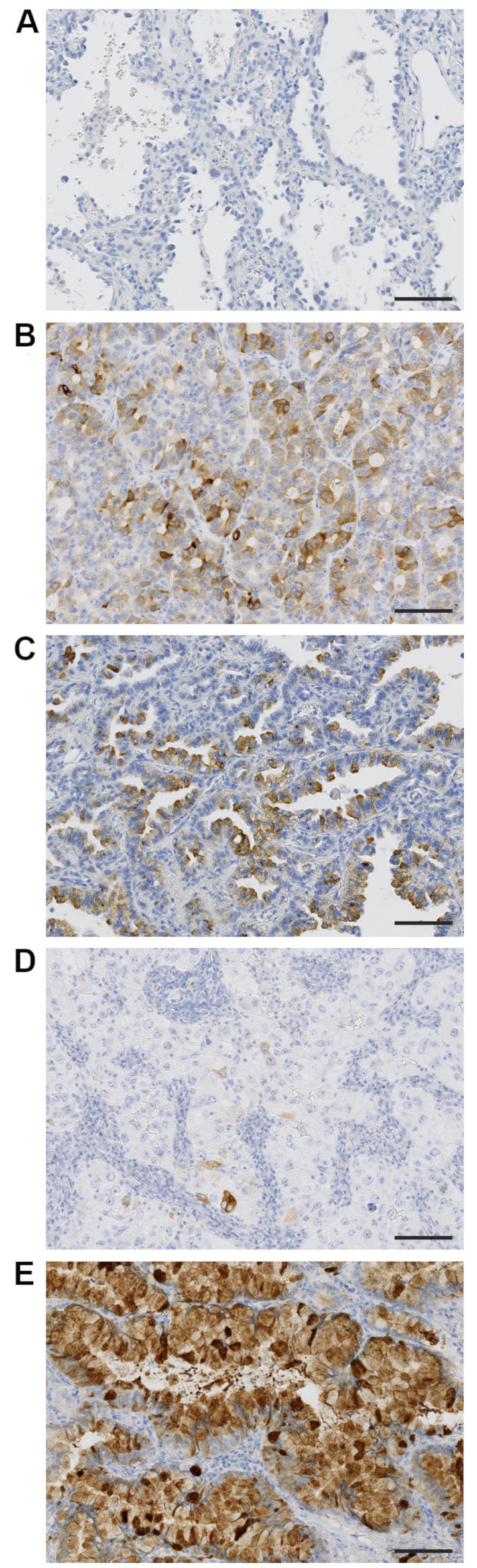

Figure 2. Trefoil factor 3 expression in various lung adenocarcinoma histologic subtypes. Typical images were presented in (A) lepidic adenocarcinoma, (B) acinar adenocarcinoma, (C) papillary adenocarcinoma, (D) solid adenocarcinoma and (E) invasive mucinous adenocarcinoma. Scale bars, $100 \mu \mathrm{m}$.
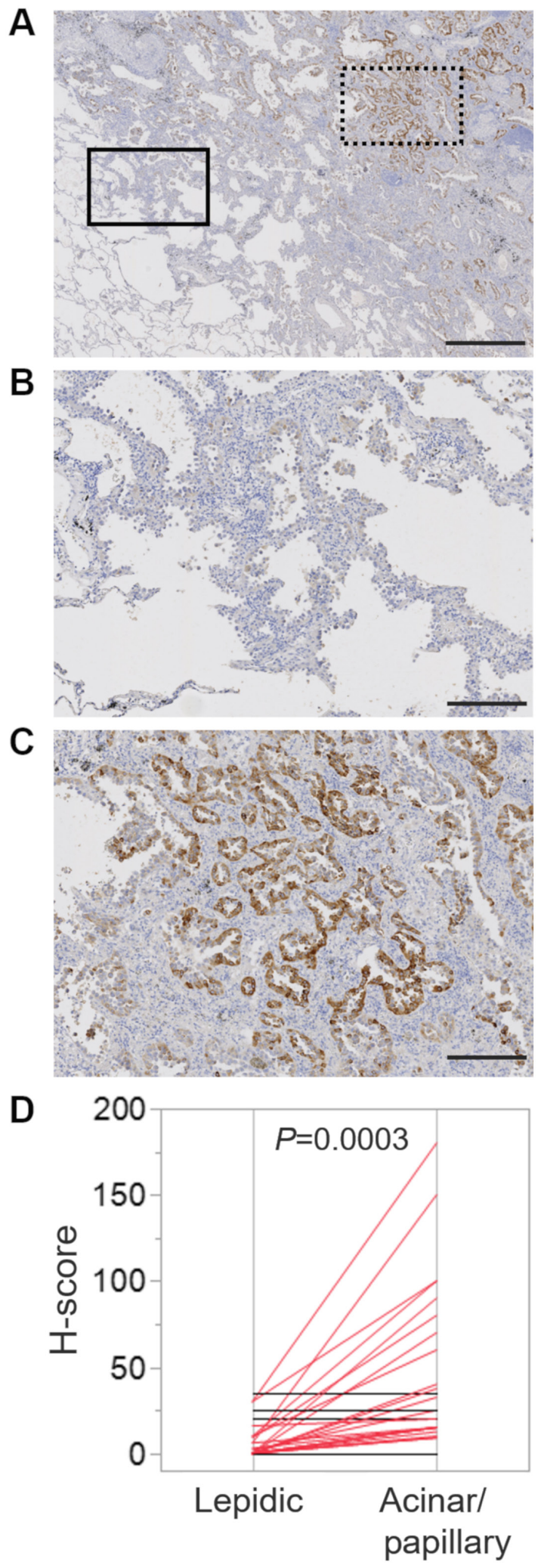

Figure 3. Varying TFF3 expression in an individual sample with mixed histologic subtypes. (A) Immunohistochemistry of TFF3 in a case with both lepidic and papillary areas. The boxed area with a solid line represents the lepidic area, which was enlarged in (B). The boxed area with a dotted line represents the papillary area, which was enlarged in (C). (D) The comparison of lepidic area and papillary/acinar area $\mathrm{H}$-scores in 38 cases with both these areas. Each line represented the $\mathrm{H}$-score of lepidic and papillary/acinar area of each case, and the data revealed a significant increase in papillary/acinar area from 22 of the 38 cases (red line). No differential expression in other samples was marked with a black line. The Wilcoxon signed-rank test was used to determine significance. Scale bar in (A), $1 \mathrm{~mm}$; Scale bars in (B an C), $250 \mu \mathrm{m}$. TTF3, trefoil factor 3 . 
A

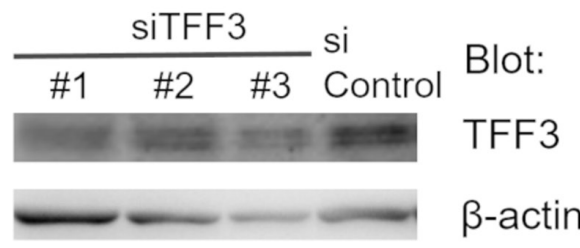

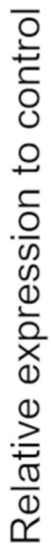
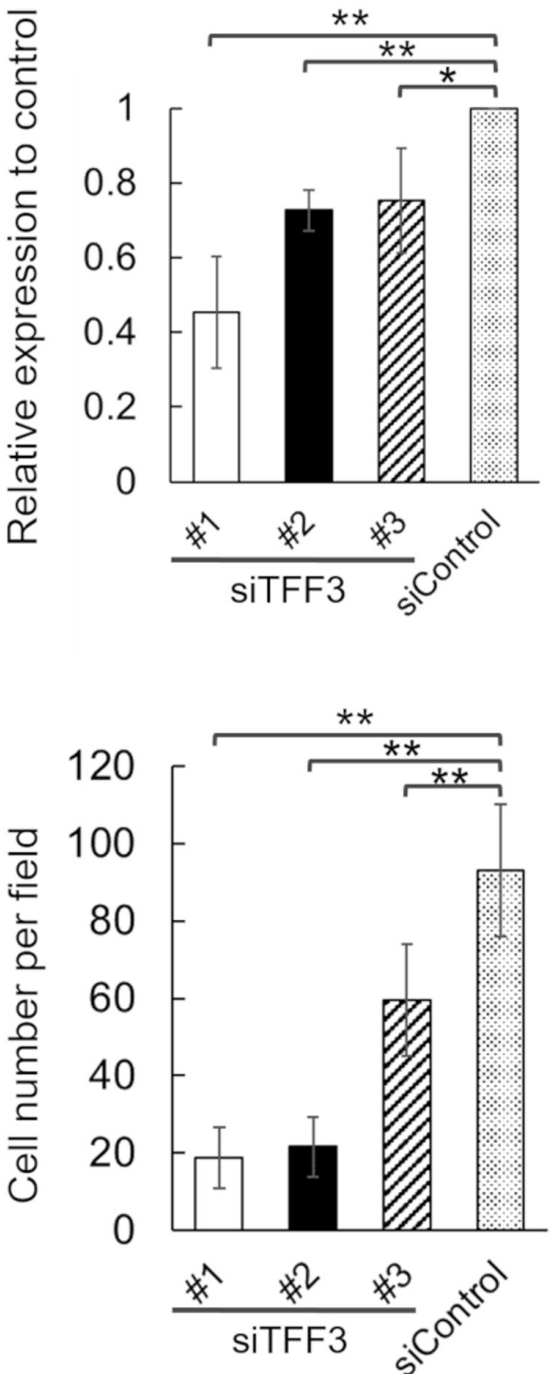

Figure 4. The association between TFF3 expression and adenocarcinoma lung cell invasiveness. (A) Confirmation of TFF3 knockdown in siTFF3 \#1, \#2 and \#3 A549 cells. Equal protein loading was confirmed by quantifying $\beta$-actin (input control). TFF3 expression was quantified using ImageJ version 1.52 software. TFF3/ $\beta$-actin quotient of control cells is expressed as 1 . The relative quotient of TFF3-knockdown cells is presented as the ratio to that of control cells. (B) Matrigel invasion assay. Representative images of invading \#1, \#2 and \#3 and control cells are presented. Invasive cells were counted in 5 random fields of view. Data are presented as the mean \pm standard error. ${ }^{*} \mathrm{P}<0.05$ and ${ }^{* *} \mathrm{P}<0.01$, as indicated. Scale bars, $100 \mu \mathrm{m}$. TTF3, trefoil factor 3 ; si, small interfering RNA.

case with both lepidic and papillary/acinar areas, lepidic area means non-invasive and papillary/acinar area means invasive area. The expression of TFF3 in papillary/acinar area was significantly higher than that of lepidic area in an individual sample. Moreover, we showed that using lung adenocarcinoma cells, the depletion of TFF3 attenuated invasion. Therefore, we proved that TFF3 is related to invasiveness by means of both in vitro and immunohistochemical assays on clinical samples.

On the other hand, the expression of TFF3 in solid adenocarcinoma, a highly invasive histologic subtype, was not significantly high. Further investigation is necessary to detect the molecular mechanism of invasion by TFF3 in lung adenocarcinoma.

Collectively, our findings revealed that in lung adenocarcinoma TFF3 was highly expressed not only in invasive mucinous carcinoma but also in papillary and acinar adenocarcinoma. This is, to our knowledge, the first report that TFF3 expression was related to the histologic subtype in lung adenocarcinoma.

\section{Acknowledgements}

The authors would like to thank Ms. Etsuko Maeno, Ms. Takako Sawamura and Mr. Masaharu Kohara (Department of Pathology, Osaka University Graduate School of Medicine) for their technical assistance.

\section{Funding}

The present study was supported by the Japan China Sasakawa Medical Fellowship (grant no. 2019-41-16); Project MEET, Osaka University Graduate School of Medicine (grant nos. A19H034520, T17K195550 and T18K150780); The Ministry of Education, Culture, Sports, Science and Technology, Japan.

\section{Availability of data and materials}

The datasets used and/or analyzed during the present study are available from the corresponding author on reasonable request. 


\section{Authors' contributions}

EM proposed and designed the current study. KK, AK and SN selected patients and collected samples/clinical data. WL and ST conducted the experiments. ST and EM evaluated immunohistochemical data. ST and EM wrote the manuscript. WL, ST, KK, AK, SN and EM reviewed and edited the manuscript. All authors approved the submitted and published versions.

\section{Ethics approval and consent to participate}

The present study was approved by the Ethical Review Board of the Graduate School of Medicine, Osaka University (approval no. 16293). Informed consent was obtained from all study participants.

\section{Patient consent for publication}

Not applicable.

\section{Competing interests}

The authors declare that they have no competing interests.

\section{References}

1. Zappa C and Mousa SA: Non-small cell lung cancer: Current treatment and future advances. Transl Lung Cancer Res 5: 288-300, 2016.

2. Lee G, Choi ER, Lee HY, Jeong JY, Ahn JH, Kim S, Bae J, Kim HK, Choi YS, Kim J, et al: Pathologic heterogeneity of lung adenocarcinomas: A novel pathologic index predicts survival. Oncotarget 7: 70353-70363, 2016
3. Plaut AG: Trefoil peptides in the defense of the gastrointestinal tract. N Engl J Med 336: 506-507, 1997.

4. Emami S, Rodrigues S, Rodrigue CM, Le Floch N, Rivat C, Attoub S, Bruyneel E and Gespach C: Trefoil factor family (TFF) peptides and cancer progression. Peptides 25: 885-898, 2004.

5. Wiede A, Jagla W, Welte T, Köhnlein T, Busk H and Hoffmann W: Localization of TFF3, a new mucus-associated peptide of the human respiratory tract. Am J Respir Crit Care Med 159: 1330-1335, 1999.

6. Taniguchi Y, Kurokawa Y, Takahashi T, Mikami J, Miyazaki Y, Tanaka K, Makino T, Yamasaki M, Nakajima K, Mori M and Doki Y: Prognostic value of trefoil factor 3 expression in patients with gastric cancer. World J Surg 42: 3997-4004, 2018.

7. Mhawech-Fauceglia P, Wang D, Samrao D, Liu S, DuPont NC and Pejovic T: Trefoil factor family 3 (TFF3) expression and its interaction with estrogen receptor (ER) in endometrial adenocarcinoma. Gynecol Oncol 130: 174-180, 2013.

8. Pandey V, Wu ZS, Zhang M, Li R, Zhang J, Zhu T and Lobie PE: Trefoil factor 3 promotes metastatic seeding and predicts poor survival outcome of patients with mammary carcinoma. Breast Cancer Res 16: 429, 2014.

9. Wang XN, Wang SJ, Pandey V, Chen P, Li Q, Wu ZS, Wu Q and Lobie PE: Trefoil factor 3 as a novel biomarker to distinguish between adenocarcinoma and squamous cell carcinoma. Medicine (Baltimore) 94: e860, 2015.

10. Travis WD, Brambilla E, Nicholson AG, Yatabe Y, Austin JHM, Beasley MB, Chirieac LR, Dacic S, Duhig E, Flieder DB, et al: The 2015 World Health Organization classification of lung tumors: Impact of genetic, clinical and radiologic advances since the 2004 classification. J Thorac Oncol 10: 1243-1260, 2015.

11. Yatabe Y, Borczuk AC and Powell CA: Do all lung adenocarcinomas follow a stepwise progression? Lung Cancer 74: 7-11, 2011.

c) (i) $\ominus$ This work is licensed under a Creative Commons Attribution-NonCommercial-NoDerivatives 4.0 International (CC BY-NC-ND 4.0) License. 